\title{
On the need for hybrid steps in Hybrid Proximal Point methods
}

\author{
Rolando Gárciga O.* $\quad$ Alfredo Iusem ${ }^{\dagger}$ \\ B. F. Svaiter \\ Instituto de Matemática Pura e Aplicada, \\ Estrada Dona Castorina 110, \\ Rio de Janeiro, RJ, 22460-320, Brazil.
}

\begin{abstract}
We show that the hybrid steps on the Hybrid Proximal Point methods, allowing for constant relative errors, are necessary in order to ensure boundedness of the iterates, even in the optimization case. Moreover, we show that such conditions do not imply that the sequence of errors results summable "a posteriori". Keywords: proximal point

method, relative error, inexact solutions, hybrid steps.
\end{abstract}

\section{Introduction}

The Proximal Point method, whose origins can be traced back e.g. to [3], [5], [4], was presented in [6] as a method for finding zeroes of monotone operators in Hilbert spaces. The exact Proximal Point Method is defined

\footnotetext{
*(rgarciga@impa.br).

$\dagger$ (iusp@impa.br). The work of this author was partially supported by CNPq grant no. $301280 / 86$

$\ddagger$ (benar@impa.br). The work of this author was partially supported by CNPq grant no. $301200 / 93-9$
} 
as follows: Given a Hilbert space $H$ and a maximal monotone point-toset operator $T: H \rightarrow \mathcal{P}(H)$, take a bounded sequence of regularization parameters $\left\{\lambda_{k}\right\} \subset \mathbb{R}_{++}$and any initial $z^{0} \in H$, and then, given $z^{k}$, define $z^{k+1}$ as the only $z \in H$ such that

$$
0 \in T(z)+\lambda_{k}\left(z-z^{k}\right)
$$

It was proved in [6] that when $T$ has zeroes the sequence $\left\{x^{k}\right\}$ defined by (1) is weakly convergent to a zero of $T$. This convergence is not strong in general, a fact that was proved by Güler in [2], through an example in the space $\ell^{2}$ for the optimization case.

The resolvent operator $P_{k}$ of $T$, for $\lambda_{k}$ fixed, is given by

$$
P_{k}=\left(T+\lambda_{k} I\right)^{-1}
$$

Hence, the exact Proximal Point Method define the sequence $\left\{z^{k}\right\}$ by the iteration $z^{k+1}=P_{k}\left(z^{k}\right)$.

Inexact variants in Hilbert spaces were considered as early as in [6], where the iteration

$$
z^{k+1} \approx P_{k}\left(z^{k}\right)
$$

is considered. Here $\left\|z^{k+1}-P_{k}\left(z^{k}\right)\right\|=\epsilon_{k}$ is the error in the $k$-th iteration, and convergence of the sequence $\left\{z^{k}\right\}$ to a zero of $T$ is ensured under the assumption that $\sum_{k=0}^{\infty} \epsilon_{k}<\infty$. Such summability condition demands that the precision in the computations increase with the iteration index $k$.

New variations of the Proximal Point Algorithm, accepting constant relative errors, have been recently presented in [7], [8], [9]. The basic feature of these methods is to combine, in each iteration, an approximate solution of the proximal problem with a projection [7] or an extragradient step [8,9]. For this reason, these are hybrid methods (proximal-projection or proximalextragradient). In both of these hybrid methods, the proximal problem (1) is decomposed in the following equivalent system:

$$
\begin{aligned}
& v \in T(z), \\
& v+\lambda_{k}\left(z-z^{k}\right)=0 .
\end{aligned}
$$

An approximate solution of these system is used to compute the next iterate by means of a projection or an extragradient step. The error tolerance is given by a fixed $\sigma \in[0,1)$. In the Hybrid Projection-Proximal method, an 
approximate solution of the proximal system is a pair $\left(\tilde{z}^{k}, \tilde{v}^{k}\right)$ with

$$
\begin{aligned}
& \tilde{v}^{k} \in T\left(\tilde{z}^{k}\right), \\
& \tilde{v}^{k}+\lambda_{k}\left(\tilde{z}^{k}-z^{k}\right)=e^{k} \approx 0 .
\end{aligned}
$$

The pair $\left(\tilde{z}^{k}, \tilde{v}^{k}\right)$ is acceptable if

$$
\left\|e^{k}\right\| \leq \sigma \max \left\{\left\|\tilde{v}^{k}\right\|, \lambda_{k}\left\|\tilde{z}^{k}-z^{k}\right\|\right\} .
$$

Then the next iterate is obtained as the orthogonal projection of $z^{k}$ onto the hyperplane $H_{k}=\left\{z \in H:\left\langle\tilde{v}^{k}, z-\tilde{z}^{k}\right\rangle=0\right\}$, i.e.

$$
z^{k+1}=z^{k}-\frac{\left\langle\tilde{v}^{k}, z^{k}-\tilde{z}^{k}\right\rangle}{\left\|\tilde{v}^{k}\right\|^{2}} \tilde{v}^{k} .
$$

Regarding the Hybrid Extragradient-Proximal method, the inclusion $\tilde{v}^{k} \in$ $T\left(\tilde{z}^{k}\right)$ in (3), may also be relaxed, by means of an enlargement of $T$, introduced in [1]. In order to simplify our exposition, we consider only approximate solutions in the sense of (4), without relaxing the inclusion. In this case, the pair $\left(\tilde{z}^{k}, \tilde{v}^{k}\right)$ (as in (4)) is acceptable if

$$
\left\|e^{k}\right\| \leq \sigma \lambda_{k}\left\|\tilde{z}^{k}-z^{k}\right\|
$$

where again $\sigma \in[0,1)$ is a fixed (relative) error tolerance. Then the next iterate is obtained by an extragradient step,

$$
z^{k+1}=z^{k}-\lambda_{k}^{-1} \tilde{v}^{k}
$$

If $T$ has zeroes, then, sequences $\left\{z^{k}\right\}$ defined by (4),(5),(6) and (4),(7),(8) respectively, converge weakly to a zero of $T$; otherwise (i.e., if $T^{-1}(0)=\emptyset$ ) these sequences are unbounded [7,9].

The main objective of this work is to answer the following questions, concerning these new methods:

1.) Is boundedness of the iterates guaranteed without the hybrid steps?

Or, equivalently:

Does the classical PPA converge under the "relative error criteria" of the hybrid methods?

2.) In the hybrid methods, do the error measures, mainly the distances from the inexact solutions to the resolvent, i.e. $\left\|\tilde{z}^{k}-P_{k}\left(z^{k}\right)\right\|$, result summable "a posteriori"? 
It should be observed that Solodov and Svaiter gave in [7] a negative answer to question (1.), taking $T(x, y)=(x,-y)$ in $\mathbb{R}^{2}$. This operator $T$ is not a subdifferential, so that question (1.) remained open for the optimization case. We prove here that, even for the optimization case, the answer for both questions is negative, at least in infinite dimensional spaces. Our examples, presented in the next section, require a function $g$ such that the sequence $\left\{x^{k}\right\}$ generated by the exact method (1) with $T=\partial g$ satisfies

$$
\sum_{k=0}^{\infty}\left\|x^{k+1}-x^{k}\right\|=\infty .
$$

A sufficient condition for (9) to hold is that the convergence of $\left\{x^{k}\right\}$ to its limit be weak but not strong, which can occur only in infinite dimensional spaces. Thus, we take $H=\ell_{2}$ in the examples. Of course, (9) could hold also for strongly convergent sequences generated by the exact method (1), but we have found no example of such a situation. So the issue remains open for the optimization case in finite dimensional spaces.

Note that the error criterium (7) is more demanding than (5). Thus, we will use (7) in our example, which then remains valid under (5). The full statement of both methods for the optimization case (i.e. $T=\partial g$ for some proper, closed and convex $g: H \rightarrow \mathbb{R} \cup\{\infty\}$ ), is given below:

1. Choose $z^{0} \in H$.

2. Given $z^{k}$, find $\tilde{v}^{k}, \tilde{z}^{k} \in H$ such that

$$
\begin{aligned}
\tilde{v}^{k} & \in \partial g\left(\tilde{z}^{k}\right), \\
\tilde{v}^{k} & +\lambda_{k}\left(\tilde{z}^{k}-z^{k}\right)=e^{k}, \\
\left\|e^{k}\right\| & \leq \sigma \lambda_{k}\left\|\tilde{z}^{k}-z^{k}\right\| .
\end{aligned}
$$

3. Hybrid step:

$$
\begin{aligned}
& z^{k+1}=z^{k}-\frac{\left\langle\tilde{v}^{k}, z^{k}-\tilde{z}^{k}\right\rangle}{\left\|\tilde{v}^{k}\right\|^{2}} \tilde{v}^{k} . \quad \text { (Projection) } \\
& z^{k+1}=z^{k}-\lambda_{k}^{-1} \tilde{v}^{k} . \quad(\text { Extragradient })
\end{aligned}
$$




\section{Answering the two open questions}

We start with Güler's example on the existence of a function for which the exact Proximal Point algorithm converges weakly but not strongly.

Proposition 1. (Güler) There exists a proper, closed and convex function in $\ell^{2}$ which has minimizers and such that, given any bounded positive sequence $\left\{\lambda_{k}\right\}$, there exists a point $x \in$ dom $f$ for which the exact Proximal Point algorithm (1), starting at $x$, converges weakly but not strongly to a minimizer of $f$.

Proof. See [2] Corollary 5.1.

We state next our main result.

Theorem 1. There exist a Hilbert space $H$ and a proper, closed and convex function $g$ in $H$ which has minimizers and such that, given any bounded positive sequence $\left\{\lambda_{k}\right\}$, there exists a point $z \in$ dom $g$ such that

i) The inexact Proximal Point algorithm allowing for constant relative errors as in (10)-(12), without the hybrid steps, starting at z, may generate unbounded sequences of iterates.

ii) The Hybrid Proximal Point methods allowing for constant relative error in (10)-(12) and (13) or (10)-(12) and (14), starting at z, accept inexact iterates with nonsummable distances to the resolvent.

Proof. Let $f$ be the function provided by Proposition 1 and $\left\{x^{k}\right\}$ the sequence generated by the exact Proximal Point Algorithm (1) starting at point $x$, also given by Proposition 1 . Then the sequence $\left\{x^{k}\right\}$ converges weakly to some $x^{*}$, but does not converge strongly. Therefore,

$$
\sum_{k=1}^{\infty}\left\|x^{k+1}-x^{k}\right\|=\infty
$$

because otherwise the sequence $\left\{x^{k}\right\}$ would converge strongly to $x^{*}$. Since $\left\{x^{k}\right\}$ is generated by Algorithm (1), there exists a sequence $\left\{u^{k}\right\}_{k \geq 1} \in \ell^{2}$ such that for all $k$ :

$$
\begin{aligned}
& u^{k+1} \in \partial f\left(x^{k+1}\right), \\
& u^{k+1}+\lambda_{k}\left(x^{k+1}-x^{k}\right)=0 .
\end{aligned}
$$


Let us consider the product space $H=\ell^{2} \times \mathbb{R}$ provided with the 2-norm $\|\cdot\|_{2}$ (i.e. $\left.\|(x, t)\|_{2}=\left(\|x\|_{\ell^{2}}^{2}+|t|^{2}\right)^{1 / 2}\right)$. Trivially, $H$ is a Hilbert space. Define the function $g: H \rightarrow \mathbb{R} \cup\{\infty\}$ as

$$
g(x, t)=f(x),
$$

for all $(x, t) \in H$. Consequently $g$ is a proper, closed and convex function with subdifferential at any $(x, t)$ given by $\partial g(x, t)=(\partial f(x), 0)$. In particular, if $S$ is the set of minimizers of $f$, then $S \times \mathbb{R}$ is the set of minimizers of $g$, hence nonempty.

Now we are ready to prove item (i). Choose any $t^{0} \in \mathbb{R}$ and define the sequence $\left\{t^{k}\right\} \in \mathbb{R}$ as

$$
t^{k+1}=t^{k}+\frac{\sigma}{\sqrt{1-\sigma^{2}}}\left\|x^{k+1}-x^{k}\right\|=t^{0}+\frac{\sigma}{\sqrt{1-\sigma^{2}}} \sum_{n=0}^{k}\left\|x^{n+1}-x^{n}\right\| .
$$

Define also, for $k=0,1, \cdots$

$$
z^{k}=\left(x^{k}, t^{k}\right), \quad e^{k}=\lambda_{k}\left(0, t^{k+1}-t^{k}\right), \quad v^{k+1}=\left(u^{k+1}, 0\right) .
$$

Then

$$
\begin{aligned}
v^{k+1} & \in \partial g\left(z^{k+1}\right), \\
v^{k+1} & +\lambda_{k}\left(z^{k+1}-z^{k}\right)=e^{k}
\end{aligned}
$$

where (21) follows from (17), (20) from (16) and the residual $e^{k}$ satisfies

$$
\begin{aligned}
\left\|e^{k}\right\|_{2} & =\lambda_{k}\left|t^{k+1}-t^{k}\right|=\lambda_{k} \frac{\sigma}{\sqrt{1-\sigma^{2}}}\left\|x^{k+1}-x^{k}\right\| \\
& =\lambda_{k} \sigma\left(\left\|x^{k+1}-x^{k}\right\|^{2}+\left|t^{k+1}-t^{k}\right|^{2}\right)^{1 / 2}=\lambda_{k} \sigma\left\|z^{k+1}-z^{k}\right\|,
\end{aligned}
$$

using (18). It follows that the pair $\left(z^{k+1}, v^{k+1}\right)$ satisfies (10)-(12), and can be accepted as an inexact solution of the $k$-th proximal subproblem. Hence, the sequence $\left\{z^{k}\right\}$ is generated by the inexact Proximal Point Algorithm, applied to an optimization problem and using the relative error tolerance of the hybrid methods. Trivially, from (15), (18) and (19),

$$
\lim _{k \rightarrow \infty}\left\|z^{k}\right\|_{2}=\infty
$$

which proves item (i). 
For (ii) let us consider first the extragradient case. i.e. the method in (10)-(12) and (14). Through an induction argument, it can be proved that starting at $z^{0}=\left(x, t^{0}\right)$ the inexact solutions can be taken as

$$
\tilde{z}^{k}=\left(x^{k+1}, t^{0}+\frac{\sigma}{\sqrt{1-\sigma^{2}}}\left\|x^{k+1}-x^{k}\right\|\right), \tilde{v}^{k}=\left(u^{k+1}, 0\right),
$$

and the iterates as

$$
z^{k}=\left(x^{k}, t^{0}\right)
$$

Then $P_{k}\left(z^{k}\right)=z^{k+1}$ for all $k$ and

$$
\left\|\tilde{z}^{k}-P_{k}\left(z^{k}\right)\right\|=\left\|\tilde{z}^{k}-z^{k+1}\right\|=\left|\tilde{t}^{k}-t^{0}\right|=\frac{\sigma}{\sqrt{1-\sigma^{2}}}\left\|x^{k+1}-x^{k}\right\|,
$$

which implies $\sum_{k=0}^{\infty}\left\|\tilde{z}^{k}-P_{k}\left(z^{k}\right)\right\|=\infty$. Note that $z^{k+1}$ is also the projection of $z^{k}$ onto $H_{k}=\left\{z \in H:\left\langle\tilde{v}^{k}, z-\tilde{z}^{k}\right\rangle=0\right\}$, hence the same example works for the Hybrid Projection Proximal Algorithm.

Remark: Observe that in the proof of item (ii), $\left\|e^{k}\right\|=\lambda_{k}\left|\tilde{t}^{k}-t^{0}\right|$ for all $k$. Consequently

$$
\sum_{k=0}^{\infty}\left\|e^{k}\right\| \geq \underline{\lambda} \sum_{k=0}^{\infty}\left\|\tilde{z}^{k}-P_{k}\left(z^{k}\right)\right\|=\infty
$$

provided that $\lambda_{k} \geq \underline{\lambda}>0$ for all $k$, so that the residuals are not summable either.

Remark: Though the example given by Theorem (1) does not prove nonsummability of the distances of the hybrid iterates to the resolvent, because $z^{k+1}=P_{k}\left(z^{k}\right)$ for all $k$, a slight modification of it (e.g. with $g(x, t)=f(x)+$ $\left.t^{2} / 2\right)$ provides an example in which the condition $\sum_{k=0}^{\infty}\left\|z^{k+1}-P_{k}\left(z^{k}\right)\right\|<\infty$ does not hold either.

\section{References}

[1] Burachik, R.S., Iusem, A.N., Svaiter, B.F. Enlargement of monotone operators with applications to variational inequalities Set-Valued Analysis 5 (1997) 159-180. 
[2] Güler, O. On the convergence of the proximal point algorithm for convex minimization. SIAM Journal on Control and Optimization 29 (1991) 403-419.

[3] Krasnoselskii, M.A. Two observations about the method of succesive approximations. Uspekhi Matematicheskikh Nauk 10 (1955) 123-127.

[4] Martinet, B. Régularisation d'inéquations variationnelles par approximations successives. Revue Française de Informatique et Recherche Opérationnelle 4 (1970) Ser. R-3, 154-158.

[5] Moreau, J. Proximité et dualité dans un espace hilbertien. Bulletin de la Societé Mathématique de France 93 (1965) 273-299.

[6] Rockafellar, R.T. Monotone operators and the proximal point algorithm. SIAM Journal on Control and Optimization 14 (1976) 877-898.

[7] Solodov, M.V., Svaiter, B.F. A hybrid projection-proximal point algorithm. Journal of Convex Analysis 6 (1999) 59-70.

[8] Solodov, M.V., Svaiter, B.F. An inexact hybrid generalized proximal point algorithms and some new results on the theory of Bregman functions. Mathematics of Operations Research 51 (2000) 479-494.

[9] Solodov, M.V., Svaiter, B.F. An inexact hybrid extragradient-proximal point algorithm using the enlargement of a maximal monotone operator. Set-Valued Analysis 7 (1999) 323-345. 